\title{
Is the Diagnosis of Spontaneous Rupture of a Normal Spleen Valid?
}

\author{
Maj I D Crate \\ FRCS(Ed), RAMC \\ Col M J Payne \\ FRCS, L/RAMC \\ Cambridge Military Hospital, Aldershot, Hants GU11 2AN
}

\begin{abstract}
Since Atkinson first described idiopathic rupture of a normal spleen in 1874, there has been controve regarding the application of the terms "idiopathic" and "spontaneous". One such case is reported, and the validity of the description "idiopathic" or "spontaneous" discussed.
\end{abstract}

\section{Case Report}

In June 1986 a 36 year old Caucasian male was admitted to the Cambridge Military Hospital, Aldershot, with acute abdominal pain. He gave a history of sudden onset of severe abdominal pain occurring two hours prior to admission, initially epigastric in location, but later radiating to both shoulder tips. The shoulder tip pain was worse on recumbency and more marked in the left than the right shoulder. He had vomited twice and complained of faintness and sweating. There was no history of past or family illness of relevance, and no previous episodes of similar pain. Two years prior to admission he had sustained minor injuries in a road traffic accident, but as far as he was aware no serious injury, specifically no splenic injury, was suspected. There was no history or clinical signs of recent trauma.

\section{Examination}

Examination showed a pale slim individual with a pulse rate of 90 beats per minute and a blood pressure of $80 / 60 \mathrm{mmHg}$. There was tenderness and guarding in the upper half of the abdomen. Bowel sounds were normal as was rectal examination. Nasogastric aspiration showed no evidence of blood.

\section{Investigations}

Blood count, urea, electrolytes, amylase and liver function tests were all normal. Erect chest and supine abdominal radiographs were normal.

\section{Clinical Course}

A diagnosis of viscus perforation, probably duodenal, was made and urgent laparotomy performed (MJP). The abdomen was opened through an upper midline incision revealing an $1100 \mathrm{ml}$ haemoperitoneum with a large clot in the left upper quadrant. The cause was seen to be a deep rupture of the spleen and emergency splenectomy performed. The postoperative course was uneventful and the patient was discharged from hospital 6 days later. Postoperative haematological indices showed only the usual post-splenectomy pattern. Tests for infectious mononucleosis (IM Denco) were negative. Detailed virological studies were not considered sufficiently early to be of value.

\section{Pathology}

Histopathological examination of the spleen showed it to weigh 312 grams (slightly enlarged), with a lase blood clot attached to its lateral surface overlying a taar in the capsule. A further tear $5 \mathrm{cms}$ in length was present anterior to the hilum on the medial aspect of the organ. No organisation of the haematomata had occurreduat either lesion.

Microscopic examination of the spleen showed tho evidence of reticulosis or inflammatory infiltration $\frac{\bar{\Phi}}{\vec{N}}$

No evidence of previous injury, scarring or adhesigns could be found.

\section{Discussion}

Atkinson(1) first described spontaneous or idio rupture of the spleen in 1874 . Since then many have been described, and the cases for and against the existence of the condition as a surgical entity discuges.

Often only individual cases were described(2-4). and in those publications where many cases were presêfited, there was often poor case selection for the to thle "spontaneous" when referring to rupture of the sple\&్gn.

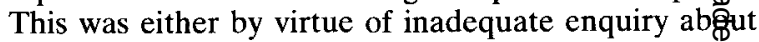
trauma, absence of reported microscopic examinatio $\underset{\rightarrow}{\rightarrow}$ the spleen, or known co-existent disease $(1,2,4,8)$.

Because of this loose application of terminology the diagnosis fell into disrepute with some auth $\overline{\text { Is }}$. Susman(9) in 1927 argued that the patholgy predisposing to the rupture was so localised that the abnormal tissue was destroyed by the ruptuite. Weber $(10)$ postulated rupture of congenital small artery aneurysms as the cause of apparent spontaneous sple rupture. One of the popular theories was that all coes reported actually represented delayed post-traumatic ruptures, and it is possible that many of the cages reported as spontaneous were indeed secondary to trauma, because often no detailed history of possiple trauma was taken.

Orloff and Peskin(11) undertook a collective reviêw of all previous cases, including their own, analysing case histories and the data presented for scrutiny. TFey found 71 reported cases, of which only 20 had 20 alternative explanation for the spleen to rupture, and identified four criteria which should be fulfilled if 至e 
diagnosis of spontaneous splenic rupture were to be sustained. These criteria were:

1) On thorough questioning either before or after operation there should be no history of trauma or unusual effort which conceivably could injure the spleen.

2) There should be no evidence of disease in organs other than the spleen which is known to affect the spleen adversely.

3) There should be no evidence of perisplenic adhesions or scarring of the spleen which suggest that it had been traumatised or had ruptured previously.

4) Other than the findings of haemorrhage and rupture, the spleen sould be normal on both macroscopic and microscopic examination.

Since 1958 many cases of "spontaneous" rupture of the spleen associated with other conditions such as leukaemia(12-16) have been reported, however the application of the term "spontaneous" to these cases is of course inaccurate according to the above criteria.

It is recognised that many viral infections affect the spleen, and the histologic changes, if any, may be delayed. It is therefore possible that a sub-clinical viraemia was responsible for the splenic rupture in our patient, and that recent viral infection as shown by rising antibody titres between the acute and convalescent stages might explain many previous cases of "spontaneous" rupture of the spleen.

We suggest that a fifth criterion may be added to Orloff and Peskin's original four, and that should be:

"Full virological studies of acute phase and convalescent sera should show no significant rise in antibody titres suggesting recent viral infection of types known to be associated with splenic involvement."

It is however very unlikely that blood would be drawn for such studies prior to emergency laparotomy. It is certain that the result would have no effect on the immediate surgical management of the patient. It therefore seems that the term "spontaneous splenic rupture" is one which is becoming more difficult to apply correctly, and by frequent misuse no longer denotes a specific surgical condition.

It is also possible that many of the historical cases in the literature were in fact associated with as then unrecognised intercurrent disease. We feel the term should be abandoned in favour of a less specific term, e.g. "non-traumatic splenic rupture" which will then allow the debate on the validity and existence of the condition to be concluded.

\section{REFERENCES}

1. AtKinson E. Death from idiopathic rupture of the spleen. Br Med J 1874; 2: 403-404.

2. INGRAM A. A spontaneous rupture of the spleen in a West African J Trop Med Hyg 1921; 24: 164.

3. JoHns W A. Splenic rupture with case report. South $M \& S$ 1944; 106: 478 .

4. JONES L E. Spontaneous rupture of an apparently normal spleen. Br Med J 1944; 2: 561.

5. Chang H L. Spontaneous rupture of the spleen. Chin Med $J$ 1948; 66: 204

6. ConNors J F. Ruptured spleens, spontaneous and subcutaneous. Ann Surg 1921; 74: 1-12.

7. DUdGEON H. Spontaneous rupture of the spleen. South Med J 1941; 34: 1247-1249.

8. RANKIN L M. Rupture of the spleen from muscular action.

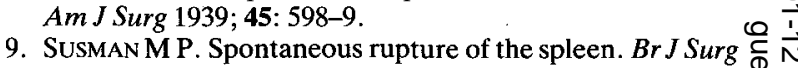
$1927 ; 15: 47-54$.

10. Weber F P. Discussion of papers by Fitzwilliams D C L. Proc Roy Soc Med 1938; 31 : 1045.

11. ORloff M J, Peskin G W. Spontaneous rupture of the normal spleen, a surgical enigma. Int Abstr Surg 1958; 106: 90.

12. Stephan P J T, Hudson P. Spontaneous rupture of the spleen in plasma cell leukaemia. Can Med Assoc J 1969; 100: 31 .

13. Rogers J S, ShaH S. Spontaneous rupture of the spleen in plasma cell leukaemia. Cancer 1980, 46: 212.

14. Karakousis C P, Elias E G. Spontaneous (pathologic) rupture of the spleen in malignancies. Surgery 1974, 76: 674.

15. Vezina W C, Nicholson R L, Cohen P, Chamberlain M J. Radionuclide diagnosis of splenic rupture in infectious mononucleosis. Clin Nucl Med 1984; 9: 341.

16. Kienzle G D, Stern J, Cooperberg A, Osborne C A. Spontaneous rupture of spleen in primary plasma cell leukaemia. Scintigraphic-pathologic correlation. Clin Nucl Med 1985; 10: 639. 